\title{
Optimization Methods for Inverse Problems
}

Nan Ye and Farbod Roosta-Khorasani and Tiangang Cui

\begin{abstract}
Optimization plays an important role in solving many inverse problems. Indeed, the task of inversion often either involves or is fully cast as a solution of an optimization problem. In this light, the mere non-linear, non-convex, and large-scale nature of many of these inversions gives rise to some very challenging optimization problems. The inverse problem community has long been developing various techniques for solving such optimization tasks. However, other, seemingly disjoint communities, such as that of machine learning, have developed, almost in parallel, interesting alternative methods which might have stayed under the radar of the inverse problem community. In this survey, we aim to change that. In doing so, we first discuss current state-of-the-art optimization methods widely used in inverse problems. We then survey recent related advances in addressing similar challenges in problems faced by the machine learning community, and discuss their potential advantages for solving inverse problems. By highlighting the similarities among the optimization challenges faced by the inverse problem and the machine learning communities, we hope that this survey can serve as a bridge in bringing together these two communities and encourage cross fertilization of ideas.
\end{abstract}

\section{Introduction}

Inverse problems arise in many applications in science and engineering. The term "inverse problem" is generally understood as the problem of finding a specific physical property, or properties, of the medium under investigation, using indirect measurements. This is a highly important field of applied mathematics and scientific computing, as to a great extent, it forms the backbone of modern science and engineering. Examples of inverse problems can be found in various fields within medical imaging $[6,7,12,71,110]$ and several areas of geophysics including mineral and oil exploration $[8,18,73,95]$.

In general, an inverse problem aims at recovering the unknown underlying parameters of a physical system which produces the available observations/measurements. Such problems are generally ill-posed [52]. This is often solved via two approaches: a Bayesian approach which computes a posterior distribution of the models given prior knowledge and the data, or a regularized data fitting approach which chooses an optimal model by minimizing an objective that takes into account both fitness to data and prior knowledge. The Bayesian approach can be used for a variety of downstream inference tasks, such as credible intervals for the parameters; it is generally more computationally expensive than the data fitting approach. The computational attractiveness of data fitting comes at a cost: it can only produce a "point" estimate of the unknown parameters. However, in many applications, such a point estimate can be more than adequate.

Nan Ye

ACEMS \& Queensland University of TechnologyInstitute, e-mail: n . yel qut . edu . au

Farbod Roosta-Khorasani

University of Queensland e-mail: fred.roostauq.edu.au

Tiangang Cui

Monash University e-mail: tiangang. cui@monash. edu 
In this review, we focus on the data fitting approach. Optimization algorithms are central in this approach as the recovery of the unknown parameters is formulated as an optimization problem. While numerous works have been done on the subject, there are still many challenges remaining, including scaling up to large-scale problems, dealing with non-convexity. Optimization constitutes a backbone of many machine learning applications [17,32]. Consequently, there are many related developments in optimization from the machine learning community. However, thus far and rather independently, the machine learning and the inverse problems communities have largely developed their own sets of tools and algorithms to address their respective optimization challenges. It only stands to reason that many of the recent advances by machine learning can be potentially applicable for addressing challenges in solving inverse problems. We aim to bring out this connection and encourage permeation of ideas across these two communities.

In Section 2, we present general formulations for the inverse problem, some typical inverse problems, and optimization algorithms commonly used to solve the data fitting problem. We discuss recent advances in optimization in Section 3. We then discuss areas in which cross-fertilization of optimization and inverse problems can be beneficial in Section 4. We conclude in Section 5.

\section{Inverse Problems}

An inverse problem can be seen as the reverse process of a forward problem, which concerns with predicting the outcome of some measurements given a complete description of a physical system. Mathematically, a physical system is often specified using a set of model parameters $\mathbf{m}$ whose values completely characterize the system. The model space $\mathscr{M}$ is the set of possible values of $\mathbf{m}$. While $\mathbf{m}$ is usually arise as a parameter function, in practice it is often discretized as a parameter vector for the ease of computation, typically using the finite element method, the finite volume method, or the finite difference method. The forward problem can be denoted as

$$
\mathbf{m} \rightarrow \mathbf{d}=\mathbf{f}(\mathbf{m}),
$$

where $\mathbf{d}$ are the error-free predictions, and the above notation is a shorthand for $\mathbf{d}=\left(\mathbf{d}_{1}, \ldots, \mathbf{d}_{s}\right)=\left(\mathbf{f}_{1}(\mathbf{m}), \ldots, \mathbf{f}_{s}(\mathbf{m})\right)$, with $\mathbf{d}_{i} \in \mathbb{R}^{l}$ being the $i$-th measurement. The function $\mathbf{f}$ represents the physical theory used for the prediction and is called the forward operator. The observed outcomes contain noises and relate to the system via the following the observation equation

$$
\mathbf{d}=\mathbf{f}(\mathbf{m})+\boldsymbol{\eta}
$$

where $\boldsymbol{\eta}$ are the noises occurred in the measurements. The inverse problem aims to recover the model parameters $\mathbf{m}$ from such noisy measurements.

The inverse problem is almost always ill-posed, because the same measurements can often be predicted by different models. There are two main approaches to deal with this issue. The Bayesian approach assumes a prior distribution $P(\mathbf{m})$ on the model and a conditional distribution $P(\boldsymbol{\eta} \mid \mathbf{m})$ on noise given the model. The latter is equivalent to a conditional distribution $P(\mathbf{d} \mid \mathbf{m})$ on measurements given the model. Given some measurements $\mathbf{d}$, a posterior distribution $P(\mathbf{m} \mid \mathbf{d})$ on the models is then computed using the Bayes rule

$$
P(\mathbf{m} \mid \mathbf{d}) \propto P(\mathbf{m}) P(\mathbf{d} \mid \mathbf{m}) .
$$

Another approach sees the inverse problem as a data fitting problem that finds an parameter vector $\mathbf{m}$ that gives predictions $\mathbf{f}(\mathbf{m})$ that best fit the observed outcomes $\mathbf{d}$ in some sense. This is often cast as an optimization problem

$$
\min _{\mathbf{m} \in \mathscr{M}} \psi(\mathbf{m}, \mathbf{d})
$$

where the misfit function $\psi$ measures how well the model $\mathbf{m}$ fits the data $\mathbf{d}$. When there is a probabilistic model of $\mathbf{d}$ given $\mathbf{m}$, a typical choice of $\psi(\mathbf{m}, \mathbf{d})$ is the negative log-likelihood. Regularization is often used to address the issue of multiple solutions, and additionally has the benefit of stabilizing the solution, that is, the solution is less likely to change significantly in the presence of outliers $[5,36,109]$. Regularization incorporats some a priori information on $\mathbf{m}$ in the form of a regularizer $R(\mathbf{m})$ and solves the regularized optimization problem 


$$
\min _{\mathbf{m} \in \mathscr{M}} \psi_{R, \alpha}(\mathbf{m}, \mathbf{d}):=\psi(\mathbf{m}, \mathbf{d})+\alpha R(\mathbf{m}),
$$

where $\alpha>0$ is a constant that controls the tradeoff between prior knowledge and the fitness to data. The regularizer $R(\mathbf{m})$ encodes a preference over the models, with preferred models having smaller $R$ values. The formulation in Eq. (5) can often be given a maximum a posteriori (MAP) interpretation within the Bayesian framework [96]. Implicit regularization also exists in which there is no explicit term $R(\mathbf{m})$ in the objective $[53,54,86,87,105,107]$.

The misfit function often has the form $\phi(\mathbf{f}(\mathbf{m}), \mathbf{d})$, which measures the difference between the prediction $\mathbf{f}(\mathbf{m})$ and the observation $\mathbf{d}$. For example, $\phi$ may be chosen to be the Euclidean distance between $\mathbf{f}(\mathbf{m})$ and $\mathbf{d}$. In this case, the regularized problem takes the form

$$
\min _{\mathbf{m} \in \mathscr{M}} \quad \phi_{R, \alpha}(\mathbf{m}, \mathbf{d}):=\phi(\mathbf{f}(\mathbf{m}), \mathbf{d})+\alpha R(\mathbf{m}),
$$

This can also be equivalently formulated as choosing the most preferred model satisfying constraints on its predictions

$$
\min _{\mathbf{m} \in \mathscr{M}} R(\mathbf{m}), \quad \text { s.t. } \quad \phi(\mathbf{f}(\mathbf{m}), \mathbf{d}) \leq \rho .
$$

The constant $\rho$ usually relates to noise and the maximum discrepancy between the measured and the predicted data, and can be more intuitive than $\alpha$.

\subsection{PDE-Contrained Inverse Problems}

For many inverse problems in science and engineering, the forward model is not given explicitly via a forward operator $\mathbf{f}(\mathbf{m})$, but often conveniently specified via a set of partial differential equations (PDEs). For such problems, Eq. (6) has the form

$$
\min _{\mathbf{m} \in \mathscr{M}, \mathbf{u}} \phi(P \cdot \mathbf{u}, \mathbf{d})+\alpha R(\mathbf{m}), \quad \text { s.t. } \quad c_{i}\left(\mathbf{m}, \mathbf{u}_{i}\right)=0, \quad i=1, \ldots, s,
$$

where $P \cdot \mathbf{u}=\left(P_{1}, \ldots, P_{s}\right) \cdot\left(\mathbf{u}_{1}, \ldots, \mathbf{u}_{s}\right)=\left(P_{1} \mathbf{u}_{1}, \ldots, P_{s} \mathbf{u}_{s}\right)$ with $\mathbf{u}_{i}$ being the field in the $i$-th experiment, $P_{i}$ being the projection operator that selects fields at measurement locations in $\mathbf{d}_{i}$ (that is, $P_{i} \mathbf{u}_{i}$ are the predicted values at locations measured in $\left.\mathbf{d}_{i}\right)$, and $c_{i}\left(\mathbf{m}, \mathbf{u}_{i}\right)=0$ corresponds to the forward model in the $i$-th experiment. In practice, the forward model can often be written as

$$
\mathscr{L}_{i}(\mathbf{m}) \mathbf{u}_{i}=\mathbf{q}_{i}, \quad i=1, \ldots, s,
$$

where $\mathscr{L}_{i}(\mathbf{m})$ is a differential operator, and $\mathbf{q}_{i}$ is a term that incorporates source terms and boundary values.

The fields $\mathbf{u}_{1}, \ldots, \mathbf{u}_{s}$ in Eq. (8) and Eq. (9) are generally functions in two or three dimensional spaces, and finding closed-form solutions is usually not possible. Instead, the PDE-constrained inverse problem is often solved numerically by discretizing Eq. (8) and Eq. (9) using the finite element method, the finite volume method, or the finite difference method. Often the discretized PDE-constrained inverse problem takes the form

$$
\min _{\mathbf{m} \in \mathscr{M}, \mathbf{u}} \phi(P \mathbf{u}, \mathbf{d})+\alpha R(\mathbf{m}), \quad \text { s.t. } \quad L_{i}(\mathbf{m}) \mathbf{u}_{i}=\mathbf{q}_{i}, \quad i=1, \ldots, s,
$$

where $P$ is a block-diagonal matrix consisting of diagonal blocks $P_{1}, \ldots, P_{s}$ representing the discretized projection operators, $\mathbf{u}$ is the concatenation of the vectors $\mathbf{u}_{1}, \ldots, \mathbf{u}_{s}$ representing the discretized fields, and each $L_{i}(\mathbf{m})$ is a square, non-singular matrix representing the differential operator $\mathscr{L}_{i}(\mathbf{m})$. Each $L_{i}(\mathbf{m})$ is typically large and sparse. We abuse the notations $P$, $\mathbf{u}$ to represent both functions and their discretized versions, but the meanings of these notations will be clear from context.

The constrained problem in Eq. (10) can be written in an unconstrained form by eliminating $\mathbf{u}$ using $\mathbf{u}_{i}=L_{i}^{-1} \mathbf{q}_{i}$,

$$
\min _{\mathbf{m} \in \mathscr{M}} \phi\left(P L^{-1}(\mathbf{m}) \mathbf{q}, \mathbf{d}\right)+\alpha R(\mathbf{m})
$$


where $L$ is the block-diagonal matrix with $L_{1}, \ldots, L_{s}$ as the diagonal blocks, and $\mathbf{q}$ is the concatenation of $\mathbf{q}_{1}, \ldots, \mathbf{q}_{s}$. Note that, as in the case of (6), here we have $\mathbf{f}(\mathbf{m})=P L^{-1}(\mathbf{m}) \mathbf{q}$.

Both the constrained and unconstrained formulations are used in practice. The constrained formulation can be solved using the method of Lagrangian multipliers. This does not require explicitly solving the forward problem as in the unconstrained formulation. However, the problem size increases, and the problem becomes one of finding a saddle point of the Lagrangian, instead of finding a minimum as in the constrained formulation.

\subsection{Image Reconstruction}

Image reconstruction studies the creation of 2-D and 3-D images from sets of 1-D projections. The 1-D projections are generally line integrals of a function representing the image to be reconstructed. In the 2-D case, given an image function $f(x, y)$, the integral along the line at a distance of $s$ away from the origin and having a normal which forms an angle $\phi$ with the $x$-axis is given by the Randon transform

$$
p(s, \phi)=\int_{-\infty}^{\infty} f(z \sin \phi+s \cos \phi,-z \cos \phi+s \sin \phi) d z .
$$

Reconstruction is often done via back projection, filtered back projection, or iterative methods [55, 76]. Back projection is the simplest but often results in a blurred reconstruction. Filtered back projection (FBP) is the analytical inversion of the Radon transform and generally yields reconstructions of much better quality than back projection. However, FBP may be infeasible in the presence of discontinuities or noise. Iterative methods generally takes the noise into account and assumes a distribution on the noise. The objective function is often chosen to be a regularized likelihood of the observation, which is then iteratively optimized using the expectation maximization (EM) algorithm.

\subsection{Objective Function}

One of the most commonly used objective function is the least squares criterion, which uses a quadratic loss and a quadratic regularizer. Assume that the noise for each experiment in (2) is independently but normally distributed, i.e., $\boldsymbol{\eta}_{i} \sim \mathscr{N}\left(0, \Sigma_{i}\right), \forall i$, where $\Sigma_{i} \in \mathbb{R}^{l \times l}$ is the covariance matrix. Let $\Sigma$ be the block-diagonal matrix with $\Sigma_{1}, \ldots, \Sigma_{s}$ as the diagonal blocks. The standard maximum likelihood (ML) approach [96], leads to minimizing the least squares (LS) misfit function

$$
\phi(\mathbf{m}):=\|\mathbf{f}(\mathbf{m})-\mathbf{d}\|_{\Sigma^{-1}}^{2}
$$

where the norm $\|x\|_{A}=\sqrt{x^{\top} A x}$ is a generalization of the Euclidean norm (assuming the matrix $A$ is positive definite, which is true in the case of $\left.\Sigma_{i}^{-1}\right)$. In the above equation, we simply write the general misfit function $\phi(\mathbf{f}(\mathbf{m}), \mathbf{d})$ as $\phi(\mathbf{m})$ by taking the measurements $\mathbf{d}$ as fixed and omitting it from the notation. As previously discussed, we often minimize a regularized misfit function

$$
\phi_{R, \alpha}(\mathbf{m}):=\phi(\mathbf{m})+\alpha R(\mathbf{m}) .
$$

The prior $R(\mathbf{m})$ is often chosen as a Gaussian regularizer $R(\mathbf{m})=\left(\mathbf{m}-\mathbf{m}_{\text {prior }}\right)^{\top} \Sigma_{m}^{-1}\left(\mathbf{m}-\mathbf{m}_{\text {prior }}\right)$. We can also write the above optimization problem as minimizing $R(\mathbf{m})$ under the constraints

$$
\sum_{i=1}^{s}\left\|\mathbf{f}_{i}(\mathbf{m})-\mathbf{d}_{i}\right\| \leq \rho .
$$

The least-squares criterion belongs to the class of $\ell_{p}$-norm criteria, which contain two other commonly used criteria: the least-absolute-values criterion and the minimax criterion [102]. These correspond to the use of the $\ell_{1}$-norm and the $\ell_{\infty}$-norm for the misfit function, while the least squares criterion uses the $\ell_{2}$-norm. Specifically, the least-absolutevalues criterion takes $\phi(\mathbf{m}):=\|\mathbf{f}(\mathbf{m})-\mathbf{d}\|_{1}$, and the minimax criterion takes $\phi(\mathbf{m}):=\|\mathbf{f}(\mathbf{m})-\mathbf{d}\|_{\infty}$. More generally, 
each coordinate in the difference may be weighted. The $\ell_{1}$ solution is more robust (that is, less sensitive to outliers) than the $\ell_{2}$ solution, which is in turn more robust than the $\ell_{\infty}$ solution [25]. The $\ell_{\infty}$ norm is desirable when outliers are uncommon but the data are corrupted by uniform noise such as the quantization errors [26].

Besides the $\ell_{2}$ regularizer discussed above, the $\ell_{1}$-norm is often used too. The $\ell_{1}$ regularizer induces sparsity in the model parameters, that is, heavier $\ell_{1}$ regularization leads to fewer non-zero model parameters.

\subsection{Optimization Algorithms}

Various optimization techniques can be used to solve the regularized data fitting problem. We focus on iterative algorithms for nonlinear optimization below as the objective functions are generally nonlinear. In some cases, the optimization problem can be transformed to a linear program. For example, linear programming can be used to solve the least-absolute-values criterion or the minimax criterion. However, linear programming are considered to have no advantage over gradient-based methods (see Section 4.4.2 in [102]), and thus we do not discuss such methods here. Nevertheless, there are still many optimization algorithms that can be covered here, and we refer the readers to [13,79].

For simplicity of presentation, we consider the problem of minimizing a function $g(\mathbf{m})$. We consider iterative algorithms which start with an iterate $\mathbf{m}_{0}$, and compute new iterates using

$$
\mathbf{m}_{k+1}=\mathbf{m}_{k}+\lambda_{k} p_{k}
$$

where $p_{k}$ is a search direction, and $\lambda_{k}$ a step size. Unless otherwise stated, we focus on unconstrained optimization. These algorithms can be used to directly solve the inverse problem in Eq. (5). We only present a selected subset of the algorithms available and have to omit many other interesting algorithms.

Newton-type methods. The classical Newton's method starts with an initial iterate $\mathbf{m}_{0}$, and computes new iterates using

$$
\mathbf{m}_{k+1}=\mathbf{m}_{k}-\left(\nabla^{2} g\left(\mathbf{m}_{k}\right)\right)^{-1} \nabla g\left(\mathbf{m}_{k}\right),
$$

that is, the search direction is $p_{k}=-\left(\nabla^{2} g\left(\mathbf{m}_{k}\right)\right)^{-1} \nabla g\left(\mathbf{m}_{k}\right)$, and the step length is $\lambda_{k}=1$. The basic Newton's method has quadratic local convergence rate at a small neighborhood of a local minimum. However, computing the search direction $p_{k}$ can be very expensive, and thus many variants have been developed. In addition, in non-convex problems, classical Newton direction might not exist (if the Hessian matrix is not invertible) or it might not be an appropriate direction for descent (if the Hessian matrix is not positive definite).

For non-linear least squares problems, where the objective function $g(\mathbf{m})$ is a sum of squares of nonlinear functions, the Gauss-Newton (GN) method is often used [99]. Extensions to more general objective functions as in Eq. (13) with covariance matrix $\Sigma$ and arbitrary regularization as in Eq. (14) is considered in [92]. Without loss of generality, assume $g(\mathbf{m})=\sum_{i=1}^{s}\left(\mathbf{f}_{i}(\mathbf{m})-\mathbf{d}_{i}\right)^{2}$. At iteration $k$, the GN search direction $p_{k}$ is given by

$$
\left(\sum_{i=1}^{s} J_{i}^{\top} J_{i}\right) p_{k}=-\nabla g
$$

where the sensitivity matrix $J_{i}$ and the gradient $\nabla g$ are given by

$$
\begin{aligned}
J_{i} & =\frac{\partial \mathbf{f}_{i}}{\partial \mathbf{m}}\left(\mathbf{m}_{k}\right), \quad i=1, \ldots, s \\
\nabla g & =2 \sum_{i=1}^{s} J_{i}^{T}\left(\mathbf{f}_{i}\left(\mathbf{m}_{k}\right)-\mathbf{d}_{i}\right)
\end{aligned}
$$

The Gauss-Newton method can be seen as an approximation of the basic Newton's method obtained by replacing $\nabla^{2} g$ by $\sum_{i=1}^{s} J_{i}^{\top} J_{i}$. The step length $\lambda_{k} \in[0,1]$ can be determined by a weak line search [79] (using, say, the Armijo algorithm starting with $\left.\lambda_{k}=1\right)$ ensuring sufficient decrease in $g\left(\mathbf{m}_{k+1}\right)$ as compared to $g\left(\mathbf{m}_{k}\right)$. 
Often several nontrivial modifications are required to adapt this prototype method for different applications, e.g., dynamic regularization $[53,86,87,106]$ and more general stabilized $G N$ studied $[31,94]$. This method replaces the solution of the linear systems defining $p_{k}$ by $r$ preconditioned conjugate gradient (PCG) inner iterations, which costs $2 r$ solutions of the forward problem per iteration, for a moderate integer value $r$. Thus, if $K$ outer iterations are required to obtain an acceptable solution then the total work estimate (in terms of the number of PDE solves) is approximated from below by $2(r+1) K s$.

Though Gauss-Newton is arguable the method of choice within the inverse problem community, other Newton-type methods exist which have been designed to suitably deal with the non-convex nature of the underlying optimization problem include Trust Region [27,112] and the Cubic Regularization [23,112]. These methods have recently found applications in machine learning [113]. Studying the advantages/disadvantages of these non-convex methods for solving inverse problems can be indeed a useful undertaking.

Quasi-Newton methods. An alternative method to the above Newton-type methods is the quasi-Newton variants including the celebrated limited memory BFGS (L-BFGS) [68,78]. BFGS iteration is closely related to conjugate gradient (CG) iteration. In particular, BFGS applied to a strongly convex quadratic objective, with exact line search as well as initial Hessian $P$, is equivalent to preconditioned $C G$ with preconditioner $P$. However, as the objective function departs from being a simple quadratic, the number of iterations of L-BFGS could be significantly higher than that of GN or trust region. In addition, it has been shown that the performance of BFGS and its limited memory version is greatly negatively affected by the high degree if ill-conditioning present in such problems $[90,91,114]$. These two factor are among the main reasons why BFGS (and L-BFGS) can be less effective compared with other Newton-type alternatives in many inversion applications [44].

Krylov subspace method. A Krylov subspace method iteratively finds the optimal solution to an optimization in a larger subspace by making use of the previous solution in a smaller subspace. One of the most commonly used Krylov subspace method is the conjugate gradient $(\mathrm{CG})$ method. CG was originally designed to solve convex quadratic minimization problems of the form $g(\mathbf{m})=\frac{1}{2} \mathbf{m}^{\top} A \mathbf{m}-b^{\top} \mathbf{m}$. Equivalently, this solves the positive definite linear system $A \mathbf{m}=b$. It computes a sequence of iterates $\mathbf{m}_{0}, \mathbf{m}_{1}, \ldots$ converging to the minimum through the following two set of equations.

$$
\begin{aligned}
& \mathbf{m}_{0}=0, \quad r_{0}=b, \quad p_{0}=r_{0}, \\
& \mathbf{m}_{k+1}=\mathbf{m}_{k}+\frac{\left\|r_{k}\right\|_{2}^{2}}{p_{k}^{\top} A p_{k}} p_{k}, \quad r_{k+1}=r_{k}-\frac{\left\|r_{k}\right\|_{2}^{2}}{p_{k}^{\top} A p_{k}} A p_{k}, \quad p_{k+1}=r_{k+1}+\frac{\left\|r_{k+1}\right\|_{2}^{2}}{\left\|r_{k}\right\|_{2}^{2}} p_{k}, \quad k \geq 0 .
\end{aligned}
$$

This can be used to solve the forward problem of the form $L_{i}(\mathbf{m}) \mathbf{u}_{i}=\mathbf{q}_{i}$, provided that $L_{i}(\mathbf{m})$ is positive definite, which is true in many cases.

CG can be used to solve the linear system for the basic Newton direction. However, the Hessian is not necessarily positive definite and modification is needed [79].

In general, $C G$ can be generalized to minimize a nonlinear function $g(\mathbf{m})[28,39]$. It starts with an arbitrary $\mathbf{m}_{0}$, and $p_{1}=-\nabla g\left(\mathbf{m}_{0}\right)$, and computes a sequence of iterates $\mathbf{m}_{1}, \mathbf{m}_{2}, \ldots$ using the equations below: for $k \geq 0$,

$$
\begin{aligned}
\mathbf{m}_{k+1} & =\arg \min _{\mathbf{m} \in\left\{\mathbf{m}_{k}+\lambda p_{k}, \lambda \in \mathbb{R}\right\}} g(\mathbf{m}), \\
p_{k+1} & =-\nabla g\left(\mathbf{m}_{k+1}\right)+\beta_{k} p_{k}, \quad \text { where } \beta_{k}=\frac{\left\|\nabla g\left(\mathbf{m}_{k+1}\right)\right\|_{2}^{2}}{\left\|\nabla g\left(\mathbf{m}_{k}\right)\right\|_{2}^{2}} .
\end{aligned}
$$

The above formula for $\beta_{k}$ is known as the Fletcher-Reeves formula. Other choices of $\beta_{k}$ exist. The following two formula are known as the Polak-Ribiere and Hestenes-Stiefel formula respectively.

$$
\begin{aligned}
& \beta_{k}=\frac{\left\langle\nabla g\left(\mathbf{m}_{k+1}\right)-\nabla g\left(\mathbf{m}_{k}\right), \nabla g\left(\mathbf{m}_{k+1}\right)\right\rangle}{\left\|\nabla g\left(\mathbf{m}_{k}\right)\right\|_{2}^{2}}, \\
& \beta_{k}=\frac{\left\langle\nabla g\left(\mathbf{m}_{k+1}\right)-\nabla g\left(\mathbf{m}_{k}\right), \nabla g\left(\mathbf{m}_{k+1}\right)\right\rangle}{p_{k}^{\top}\left(\nabla g\left(\mathbf{m}_{k+1}\right)-\nabla g\left(\mathbf{m}_{k}\right)\right)} .
\end{aligned}
$$


In practice, nonlinear CG does not seem to work well, and is mainly used together with other methods, such as in the Newton CG method [79].

Lagrangian method of multipliers. The above discussion focuses on unconstrained optimization algorithms, which are suitable for unconstrained formulations of inverse problems, or unconstrained auxiliary optimization problems in methods which solves the constrained formulations directly. The Lagrangian method of multipliers is often used to directly solve the constrained version. Algorithms have been developed to offset the heavier computational cost and slow convergence rates of standard algorthms observed on the Lagrangian, which is a larger problem than the constrained problem. For example, such algorithm may reduce the problem to a smaller one, such as working with the reduced Hessian of the Lagrangian [49], or preconditioning [10,48]. These methods have shown some success in certain PDE-constrained optimization problems.

Augmented Lagrangian methods have also been developed (e.g. [1,57]). These methods constructs a series of penalized Lagrangians with vanishing penalty, and finds an optimizer of the Lagrangian by successively optimizing the penalized Lagrangians.

\subsection{Challenges}

Scaling up to large problems. The discretized version of an inverse problem is usually of very large scale, and working with fine resolution or discretized problems in high dimension is still an active area of research.

Another challenge is to scale up to large number of measurements, which is widely believed to be helpful for quality reconstruction of the model in practice, with some theoretical support. While recent technological advances makes many big datasets available, existing algorithms cannot efficiently cope with such datasets. Examples of such problems include electromagnetic data inversion in mining exploration $[33,45,77,80]$, seismic data inversion in oil exploration [38,56,88], diffuse optical tomography (DOT) [6, 14], quantitative photo-acoustic tomography (QPAT) [42, 116], direct current (DC) resistivity [31,46, 47, 83, 98], and electrical impedance tomography (EIT) $[16,24,108]$.

It has been suggested that many well-placed experiments yield practical advantage in order to obtain reconstructions of acceptable quality. For the special case where the measurement locations as well as the discretization matrices do not change from one experiment to another, various approximation techniques have been proposed to reduce the effective number of measurements, which in turn implies a smaller scale optimization problem, under the unifying category of "simultaneous sources inversion" [50,63,89,92,94]. Under certain circumstances, even if the $P_{i}$ 's are different across experiments (but $L_{i}$ 's are fixed), there are methods to transform the existing data set into the one where all sources share the same receivers, [93].

Dealing with non-convexity. Another major source of difficulty in solving many inverse problems, is the high-degree of non-linearity and non-convexity in (1). This is most often encountered in problems involving PDE-constrained optimization where each $\mathbf{f}_{i}$ corresponds to the solution of a PDE. Even if the output of the PDE model itself, i.e., the "right-hand side", is linear in the sought-after parameter, the solution of the PDE, i.e., the forward problem, shows a great deal of non-linearity. This coupled with a great amount of non-convexity can have significant consequences in the quality of inversion and the obtained parameter. Indeed, in presence of non-convexity, the large-scale computational challenges are exacerbated, multiple folds over, by the difficulty of avoiding (possibly degenerate) saddle-points as well as finding (at least) a local minimum.

Dealing with discontinuity. While the parameter function of the model is often smooth, the parameter function can be discontinuous in some cases. Such discontinuities arise very naturally as a result of the physical properties of the underlying physical system, e.g., EIT and DC resistivity, and require non-trivial modifications to optimization algorithms, e.g., [31,94]. Ignoring such discontinuities can lead to unsatisfactory recovery results $[30,31,101]$. The level set method [82] is often used to model discontinuous parameter function. This reparametrizes the discontinuous parameter function as a differentiable one, and thus enabling more stable optimization [30]. 


\section{Recent Advances in Optimization}

Recent successes in using machine learning to deal with challenging perception and natural language understanding problems have spurred many advances in the study of optimization algorithms as optimization is a building block in machine learning. These new developments include efficient methods for large-scale optimization, methods designed to handle non-convex problems, methods incorporating the structural constraints, and finally the revival of secondorder methods. While these developments address a different set of applications in machine learning, they address similar issues as encountered in inverse optimization and could be useful. We highlight some of the works below. We keep the discussion brief because numerous works have been done behind these developments and an indepth and comprehensive discussion is beyond the scope of this review. Our objective is thus to delineate the general trends and ideas, and provide references for interested readers to dig on relevant topics.

Stochastic optimization. The development in large-scale optimization methods is driven by the availability of many large datasets, which are made possible by the rapid development and extensive use of IT technology. In machine learning, a model is generally built by optimizing a sum of misfit on the examples. This finite-sum structure naturally invites the application of stochastic optimization algorithms. This is mainly due to the fact that stochastic algorithms recover the sought-after models more efficiently by employing small batches of data in each iteration, as opposed to the whole data-set. The most well-known stochastic gradient based algorithm is the stochastic gradient descent (SGD). To minimize a finite-sum objective function

$$
g(\mathbf{m})=\frac{1}{n} \sum_{i=1}^{n} g_{i}(\mathbf{m})
$$

in the big data regime where $n \gg 1$, the vanilla SGD performs an update

$$
\mathbf{m}_{k+1}=\mathbf{m}_{k}-\lambda_{k} \nabla g_{i_{k}}\left(\mathbf{m}_{k}\right),
$$

where $i_{k}$ is randomly sampled from $1, \ldots, n$. As compared to gradient descent, SGD replaces the full gradient $\nabla g(\mathbf{m})$ by a stochastic gradient $g_{i_{k}}\left(\mathbf{m}_{k}\right)$ with its expectation being the full gradient. The batch version of SGD constructs a stochastic gradient by taking the average of several stochastic gradients.

SGD is inexpensive per iteration, but suffers from a slow rate of convergence. For example, while full gradient descent achieves a linear convergence rate for smooth strongly convex problems, SGD only converges at a sublinear rate. The slow convergence rate can be partly accounted by the variance in the stochastic gradient. Recently, variance reduction techniques have been developed, e.g. SVRG [61] and SDCA [97]. Perhaps surprisingly, such variants can achieve linear convergence rates on convex smooth problems as full gradient descent does, instead of sublinear rates achieved by the vanilla SGD. There are also a number of variants with no known linear rates but have fast convergence rates for non-convex problems in practice, e.g., AdaGrad [34], RMSProp [103], ESGD [29], Adam [62], and Adadelta [117]. Indeed, besides efficiency, stochastic optimization algorithms also seem to be able to cope with the nonconvex objective functions well, and play a key role in the revival of neural networks as deep learning $[43,60,66]$.

Nonconvex optimization. There is also an increasing interest in non-convex optimization in the machine learning community recently. Nonconvex objectives not only naturally occur in deep learning, but also occur in problems such as tensor decomposition, variable selection, low-rank matrix completion, e.g. see $[43,59,72]$ and references therein.

As discussed above, stochastic algorithms have been found to be capable of effectively escaping local minima. There are also a number of studies which adapt well-known acceleration techniques for convex optimization to accelerate the convergence rates of both stochastic and non-stochastic optimization algorithms for nonconvex problems, e.g., $[4,67,85,100]$.

Dealing with structural constraints. Many problems in machine learning come with complex structural constraints. The Frank-Wolfe algorithm (a.k.a. conditional gradient) [41] is an algorithm for optimizing over a convex domain. It has gained a revived interest due to its ability to deal with many structural constraints efficiently. It requires solving a linear minimization problem over the feasible set, instead of a quadratic program as in the case of proximal gradient algorithms or projected gradient descent. Domains suitable for the Frank-Wolfe algorithm include simplices, $\ell_{p}$-balls, matrix nuclear norm ball, matrix operator norm ball [58]. 
The Frank-Wolfe algorithm belongs to the class of linear-optimization-based algorithms [64,65]. These algorithms share with the Frank-Wolfe algorithm the characteristic of requiring a first-order oracle for gradient computation and an oracle for solving a linear optimization problem over the constraint set.

Second-order methods. The great appeal of the second-order methods lies mainly in the observed empirical performance as well as some very appealing theoretical properties. For example, it has been shown that stochastic Newtontype methods in general, and Gauss-Newton in particular, can not only be made scalable and have low per-iteration cost $[31,49,51,92,93,94]$, but more importantly, and unlike first-order methods, are very resilient to many adversarial effects such as ill-conditioning [90,91,114]. As a result, for moderately to very ill-conditioned problems, commonly found in scientific computing, while first-order methods make effectively no progress at all, second-order counterparts are not affected by the degree of ill-conditioning. A more subtle, yet potentially more severe draw-back in using first-order methods, is that their success is tightly intertwined with fine-tunning (often many) hyper-parameters, most importantly, the step-size [11]. In fact, it is highly unlikely that many of these methods exhibit acceptable performance on first try, and it often takes many trials and errors before one can see reasonable results. In contrast, second-order optimization algorithms involve much less parameter tuning and are less sensitive to the choice of hyperparameters $[11,113]$.

Since for the finite-sum problem (27) with $n \gg 1$, the operations with the Hessian/gradient constitute major computational bottlenecks, a rather more recent line of research is to construct the inexact Hessian information using the application of randomized methods. Specifically, for convex optimization, the stochastic approximation of the full Hessian matrix in the classical Newton's method has been recently considered in $[3,11,15,19,20,35,37,74,75,84$, $90,91,111,114,115]$. In addition to inexact Hessian, a few of these methods study the fully stochastic case in which the gradient is also approximated, e.g., $[15,90,91]$. For non-convex problems, however, the literature on methods that employ randomized Hessian approximation is significantly less developed than that of convex problems. A few recent examples include the stochastic trust region [112], stochastic cubic regularization [104,112], and noisy negative curvature method [69]. Empirical performance of many of these methods for some non-convex machine learning applications has been considered in [113].

\section{Discussion}

Optimization is not only used in the data fitting approach to inverse problems, but also used in the Bayesian approach. An important problem in the Bayesian approach is the choice of the parameters for the prior. While these were often chosen in a somewhat ad hoc way, there are studies which use sampling [2,40], hierarchical prior models [21,22], and optimization $[9,70]$ methods to choose the parameters. While choosing the prior parameters through optimization has found some success, such optimization is hard and it remains a challenge to develop effective algorithms to solve these problems.

For inverse problems with large number of measurements, solving each forward problem can be expensive, and the mere evaluation of the misfit function may become computationally prohibitive. Stochastic optimization algorithms might be beneficial in this case, because the objective function is often a sum of misfits over different measurements.

The data fitting problem is generally non-convex and thus optimization algorithms may be trapped in a local optimum. Stochastic optimization algorithms also provide a means to escape the local optima. Recent results in nonconvex optimization, such as those on accelerated methods, may provide more efficient alternatives to solve the data fitting problem.

While box constraints are often used in inverse problems because they are easier to deal with, simplex constraint can be beneficial. The Frank-Wolfe algorithm provides a efficient way to deal with the simplex constraint, and can be a useful tool to add on to the toolbox of an inverse problem researcher. 


\section{Conclusion}

The state-of-the-art optimization methods used in the inverse problem community do not yet cope with some challenges, such as large-scale problems, nonconvexity, very well. At the same time, many progresses in optimization have been made in the machine learning community. While our discussion on the connections are nevertheless brief, we hope it has pointed out some interesting avenues that the two communities can learn from each other and work together.

\section{References}

1. Gassan S Abdoulaev, Kui Ren, and Andreas H Hielscher. Optical tomography as a PDE-constrained optimization problem. Inverse Problems, 21(5):1507 - 1530, 2005.

2. Sergios Agapiou, Johnathan M Bardsley, Omiros Papaspiliopoulos, and Andrew M Stuart. Analysis of the gibbs sampler for hierarchical inverse problems. SIAM/ASA Journal on Uncertainty Quantification, 2(1):511-544, 2014.

3. Naman Agarwal, Brian Bullins, and Elad Hazan. Second order stochastic optimization in linear time. arXiv preprint arXiv:1602.03943, 2016.

4. Zeyuan Allen-Zhu and Elad Hazan. Variance reduction for faster non-convex optimization. arXiv preprint arXiv:1603.05643, 2016.

5. G. Archer and DM. Titterington. On some bayesian/regularization methods for image restoration. Image Processing, IEEE Transactions on, 4(7):989-995, 1995.

6. S. R. Arridge. Optical tomography in medical imaging. Inverse problems, 15(2):R41, 1999.

7. S. R. Arridge and J. C. Hebden. Optical imaging in medicine: Ii. modelling and reconstruction. Physics in Medicine and Biology, 42(5):841, 1997.

8. R. C. Aster, B. Borchers, and C. H. Thurber. Parameter estimation and inverse problems. Academic Press, 2013.

9. Johnathan M Bardsley, Daniela Calvetti, and Erkki Somersalo. Hierarchical regularization for edge-preserving reconstruction of pet images. Inverse Problems, 26(3):035010, 2010.

10. Michele Benzi, Eldad Haber, and Lauren Taralli. A preconditioning technique for a class of pde-constrained optimization problems. Advances in Computational Mathematics, 35(2):149-173, 2011.

11. Albert S Berahas, Raghu Bollapragada, and Jorge Nocedal. An Investigation of Newton-Sketch and Subsampled Newton Methods. arXiv preprint arXiv:1705.06211, 2017

12. M. Bertero and P. Boccacci. Introduction to inverse problems in imaging. CRC press, 2010.

13. Åke Björck. Numerical methods for least squares problems. SIAM, 1996.

14. D.A. Boas, D.H. Brooks, E.L. Miller, C. A. DiMarzio, M. Kilmer, R.J. Gaudette, and Q. Zhang. Imaging the body with diffuse optical tomography. Signal Processing Magazine, IEEE, 18(6):57-75, 2001.

15. Raghu Bollapragada, Richard Byrd, and Jorge Nocedal. Exact and inexact subsampled Newton methods for optimization. arXiv preprint arXiv:1609.08502, 2016.

16. L. Borcea, J. G. Berryman, and G. C. Papanicolaou. High-contrast impedance tomography. Inverse Problems, 12:835-858, 1996.

17. Léon Bottou, Frank E Curtis, and Jorge Nocedal. Optimization methods for large-scale machine learning. arXiv preprint arXiv:1606.04838, 2016.

18. C. Bunks, F. M. Saleck, S. Zaleski, and G. Chavent. Multiscale seismic waveform inversion. Geophysics, 60(5):1457-1473, 1995.

19. Richard H. Byrd, Gillian M. Chin, Will Neveitt, and Jorge Nocedal. On the use of stochastic Hessian information in optimization methods for machine learning. SIAM Journal on Optimization, 21(3):977-995, 2011.

20. Richard H. Byrd, Gillian M. Chin, Jorge Nocedal, and Yuchen Wu. Sample size selection in optimization methods for machine learning. Mathematical programming, 134(1):127-155, 2012

21. Daniela Calvetti and Erkki Somersalo. A gaussian hypermodel to recover blocky objects. Inverse problems, 23(2):733, 2007.

22. Daniela Calvetti and Erkki Somersalo. Hypermodels in the bayesian imaging framework. Inverse Problems, 24(3):034013, 2008.

23. Coralia Cartis, Nicholas IM Gould, and Philippe L Toint. Evaluation complexity of adaptive cubic regularization methods for convex unconstrained optimization. Optimization Methods and Software, 27(2):197-219, 2012.

24. M. Cheney, D. Isaacson, and J. C. Newell. Electrical impedance tomography. SIAM Review, 41:85-101, 1999.

25. Jon F Claerbout and Francis Muir. Robust modeling with erratic data. Geophysics, 38(5):826-844, 1973.

26. Christian Clason. Lo fitting for inverse problems with uniform noise. Inverse Problems, 28(10):104007, 2012.

27. Andrew R Conn, Nicholas IM Gould, and Ph L Toint. Trust region methods, volume 1. SIAM, 2000.

28. Y. Dai. Nonlinear conjugate gradient methods. Wiley Encyclopedia of Operations Research and Management Science, 2011.

29. Yann Dauphin, Harm de Vries, and Yoshua Bengio. Equilibrated adaptive learning rates for non-convex optimization. In Advances in Neural Information Processing Systems, pages 1504-1512, 2015.

30. K. van den Doel, U. Ascher, and A. Leitao. Multiple level sets for piecewise constant surface reconstruction in highly ill-posed problems. Journal of Scientific Computation, 43(1):44-66, 2010.

31. Kees van den Doel and Uri Ascher. Adaptive and stochastic algorithms for EIT and DC resistivity problems with piecewise constant solutions and many measurements. SIAM J. Scient. Comput., 34:DOI: 10.1137/110826692, 2012. 
32. Pedro Domingos. A few useful things to know about machine learning. Communications of the ACM, 55(10):78-87, 2012.

33. O. Dorn, E. L. Miller, and C. M. Rappaport. A shape reconstruction method for electromagnetic tomography using adjoint fields and level sets. Inverse Problems, 16, 2000. 1119-1156.

34. John Duchi, Elad Hazan, and Yoram Singer. Adaptive subgradient methods for online learning and stochastic optimization. The Journal of Machine Learning Research, 12:2121-2159, 2011.

35. Mark Eisen, Aryan Mokhtari, and Alejandro Ribeiro. Large Scale Empirical Risk Minimization via Truncated Adaptive Newton Method. arXiv preprint arXiv:1705.07957, 2017.

36. H. W. Engl, M. Hanke, and A. Neubauer. Regularization of Inverse Problems. Kluwer, Dordrecht, 1996.

37. Murat A. Erdogdu and Andrea Montanari. Convergence rates of sub-sampled newton methods. In Advances in Neural Information Processing Systems 28, pages 3034-3042. 2015.

38. A. Fichtner. Full Seismic Waveform Modeling and Inversion. Springer, 2011.

39. R. Fletcher. Practical methods of optimization. John Wiley \& Sons, 2013.

40. Colin Fox and Richard A Norton. Fast sampling in a linear-gaussian inverse problem. SIAM/ASA Journal on Uncertainty Quantification, 4(1):1191-1218, 2016.

41. Marguerite Frank and Philip Wolfe. An algorithm for quadratic programming. Naval research logistics quarterly, 3(1-2):95-110, 1956.

42. H. Gao, S. Osher, and H. Zhao. Quantitative photoacoustic tomography. In Mathematical Modeling in Biomedical Imaging II, pages 131-158. Springer, 2012.

43. Rong Ge, Furong Huang, Chi Jin, and Yang Yuan. Escaping from saddle points-online stochastic gradient for tensor decomposition. In COLT, pages 797-842, 2015.

44. E Haber. Quasi-newton methods for large-scale electromagnetic inverse problems. Inverse problems, 21(1):305, 2004.

45. E. Haber, U. Ascher, and D. Oldenburg. Inversion of 3D electromagnetic data in frequency and time domain using an inexact all-at-once approach. Geophysics, 69:1216-1228, 2004.

46. E. Haber, M. Chung, and F. Herrmann. An effective method for parameter estimation with PDE constraints with multiple right-hand sides. SIAM J. Optimization, 22:739-757, 2012.

47. E. Haber, S. Heldmann, and U. Ascher. Adaptive finite volume method for distributed non-smooth parameter identification. Inverse Problems, 23:1659-1676, 2007.

48. Eldad Haber and Uri M Ascher. Preconditioned all-at-once methods for large, sparse parameter estimation problems. Inverse Problems, 17(6): 1847, 2001.

49. Eldad Haber, Uri M. Ascher, and Doug Oldenburg. On optimization techniques for solving nonlinear inverse problems. Inverse problems, 16(5):1263, 2000.

50. Eldad Haber and Mathias Chung. Simultaneous source for non-uniform data variance and missing data. arXiv preprint arXiv:1404.5254, 2014.

51. Eldad Haber, Matthias Chung, and Felix Herrmann. An effective method for parameter estimation with PDE constraints with multiple right-hand sides. SIAM Journal on Optimization, 22(3):739-757, 2012.

52. Jacques Hadamard. Sur les problèmes aux dérivées partielles et leur signification physique. Princeton University Bulletin, pages 49 $-52,1902$.

53. M. Hanke. Regularizing properties of a truncated Newton-cg algorithm for nonlinear inverse problems. Numer. Funct. Anal. Optim., 18:971-993, 1997.

54. P. C. Hansen. Rank-Deficient and Discrete Ill-Posed Problems. SIAM, 1998.

55. Gabor T Herman. Fundamentals of computerized tomography: image reconstruction from projections. Springer Science \& Business Media, 2009.

56. F. Herrmann, Y. Erlangga, and T. Lin. Compressive simultaneous full-waveform simulation. Geophysics, 74:A35, 2009.

57. Kazufumi Ito and Karl Kunisch. The augmented lagrangian method for parameter estimation in elliptic systems. SIAM Journal on Control and Optimization, 28(1):113-136, 1990.

58. Martin Jaggi. Revisiting Frank-Wolfe: Projection-free sparse convex optimization. In Proceedings of the 30th International Conference on Machine Learning (ICML-13), pages 427-435, 2013.

59. Prateek Jain, Praneeth Netrapalli, and Sujay Sanghavi. Low-rank matrix completion using alternating minimization. In Proceedings of the forty-fifth annual ACM symposium on Theory of computing, pages 665-674. ACM, 2013.

60. Chi Jin, Rong Ge, Praneeth Netrapalli, Sham M Kakade, and Michael I Jordan. How to escape saddle points efficiently. arXiv preprint arXiv:1703.00887, 2017.

61. Rie Johnson and Tong Zhang. Accelerating stochastic gradient descent using predictive variance reduction. In Advances in Neural Information Processing Systems, pages 315-323, 2013.

62. Diederik Kingma and Jimmy Ba. Adam: A method for stochastic optimization. arXiv preprint arXiv:1412.6980, 2014.

63. R. Kumar, C. Da Silva, O. Akalin, A. Y. Aravkin, H. Mansour, B. Recht, and F. J. Herrmann. Efficient matrix completion for seismic data reconstruction. Submitted to Geophysics on August 8, 2014., 082014.

64. Guanghui Lan, Sebastian Pokutta, Yi Zhou, and Daniel Zink. Conditional accelerated lazy stochastic gradient descent. In ICML. PMLR, 2017.

65. Guanghui Lan and Yi Zhou. Conditional gradient sliding for convex optimization. SIAM Journal on Optimization, 26(2):1379-1409, 2016.

66. Kfir Y Levy. The Power of Normalization: Faster Evasion of Saddle Points. arXiv preprint arXiv:1611.04831, 2016.

67. Huan Li and Zhouchen Lin. Accelerated proximal gradient methods for nonconvex programming. In Advances in neural information processing systems, pages 379-387, 2015. 
68. Dong C Liu and Jorge Nocedal. On the limited memory BFGS method for large scale optimization. Mathematical programming, 45(1-3):503-528, 1989.

69. Mingrui Liu and Tianbao Yang. On Noisy Negative Curvature Descent: Competing with Gradient Descent for Faster Non-convex Optimization. arXiv preprint arXiv:1709.08571, 2017.

70. Wenqing Liu, Jinglai $\mathrm{Li}$, and Youssef M Marzouk. An approximate empirical bayesian method for large-scale linear-gaussian inverse problems. arXiv preprint arXiv:1705.07646, 2017.

71. AK. Louis. Medical imaging: state of the art and future development. Inverse Problems, 8(5):709, 1992.

72. Rahul Mazumder, Jerome H Friedman, and Trevor Hastie. Sparsenet: Coordinate descent with nonconvex penalties. Journal of the American Statistical Association, 106(495):1125-1138, 2011.

73. W. Menke. Geophysical data analysis: discrete inverse theory. Academic press, 2012.

74. Mojmìr Mutnỳ. Stochastic Second-Order Optimization via von Neumann Series. arXiv preprint arXiv:1612.04694, 2016.

75. Mojmír Mutnỳ and Peter Richtárik. Parallel Stochastic Newton Method. arXiv preprint arXiv:1705.02005, 2017.

76. Frank Natterer and Frank Wübbeling. Mathematical methods in image reconstruction. SIAM, 2001.

77. G. A. Newman and D. L. Alumbaugh. Frequency-domain modelling of airborne electromagnetic responses using staggered finite differences. Geophys. Prospecting, 43:1021-1042, 1995.

78. Jorge Nocedal. Updating quasi-Newton matrices with limited storage. Mathematics of computation, 35(151):773-782, 1980.

79. Jorge Nocedal and Stephen Wright. Numerical optimization. Springer Science \& Business Media, 2006.

80. D. Oldenburg, E. Haber, and R. Shekhtman. 3D inverseion of multi-source time domain electromagnetic data. J. Geophysics, 2013. To appear.

81. S. Osher and R. Fedkiw. Level Set Methods and Dynamic Implicit Surfaces. Springer, 2003.

82. S. Osher and J. Sethian. Fronts propagating with curvature dependent speed: algorithms based on Hamilton-Jacobi formulations. $J$. Comp. Phys., 79:12-49, 1988.

83. A. Pidlisecky, E. Haber, and R. Knight. RESINVM3D: A MATLAB 3D Resistivity Inversion Package. Geophysics, 72(2):H1-H10, 2007.

84. Mert Pilanci and Martin J. Wainwright. Newton sketch: A linear-time optimization algorithm with linear-quadratic convergence. arXiv preprint arXiv:1505.02250, 2015.

85. Sashank J Reddi, Ahmed Hefny, Suvrit Sra, Barnabas Poczos, and Alex Smola. Stochastic variance reduction for nonconvex optimization. arXiv preprint arXiv:1603.06160, 2016.

86. A. Rieder. Inexact Newton regularization using conjugate gradients as inner iteration. SIAM J. Numer. Anal., 43:604-622, 2005.

87. A. Rieder and A. Lechleiter. Towards a general convergence theory for inexact Newton regularizations. Numer. Math., 114(3):521$548,2010$.

88. J. Rohmberg, R. Neelamani, C. Krohn, J. Krebs, M. Deffenbaugh, and J. Anderson. Efficient seismic forward modeling and acquisition using simultaneous random sources and sparsity. Geophysics, 75(6):WB15-WB27, 2010.

89. Farbod Roosta-Khorasani. Randomized algorithms for solving large scale nonlinear least squares problems. $\mathrm{PhD}$ thesis, University of British Columbia, 2015.

90. Farbod Roosta-Khorasani and Michael W. Mahoney. Sub-sampled Newton methods I: Globally convergent algorithms. arXiv preprint arXiv:1601.04737, 2016.

91. Farbod Roosta-Khorasani and Michael W. Mahoney. Sub-sampled Newton methods II: Local convergence rates. arXiv preprint arXiv:1601.04738, 2016

92. Farbod Roosta-Khorasani, Gábor J. Székely, and Uri Ascher. Assessing stochastic algorithms for large scale nonlinear least squares problems using extremal probabilities of linear combinations of gamma random variables. SIAM/ASA Journal on Uncertainty Quantification, 3(1):61-90, 2015.

93. Farbod Roosta-Khorasani, Kees van den Doel, and Uri Ascher. Data completion and stochastic algorithms for PDE inversion problems with many measurements. Electronic Transactions on Numerical Analysis, 42:177-196, 2014.

94. Farbod Roosta-Khorasani, Kees van den Doel, and Uri Ascher. Stochastic algorithms for inverse problems involving PDEs and many measurements. SIAM J. Scientific Computing, 36(5):S3-S22, 2014.

95. B. H. Russell. Introduction to seismic inversion methods, volume 2. Society of Exploration Geophysicists, 1988.

96. L. L. Scharf. Statistical signal processing, volume 98. Addison-Wesley Reading, MA, 1991.

97. Shai Shalev-Shwartz and Tong Zhang. Stochastic dual coordinate ascent methods for regularized loss. The Journal of Machine Learning Research, 14(1):567-599, 2013.

98. N. C. Smith and K. Vozoff. Two dimensional DC resistivity inversion for dipole dipole data. IEEE Trans. on geoscience and remote sensing, GE 22:21-28, 1984.

99. Wenyu Sun and Ya-Xiang Yuan. Optimization theory and methods: nonlinear programming, volume 1. Springer Science \& Business Media, 2006.

100. Ilya Sutskever, James Martens, George Dahl, and Geoffrey Hinton. On the importance of initialization and momentum in deep learning. In International conference on machine learning, pages 1139-1147, 2013.

101. X.-C. Tai and H. Li. A piecewise constant level set method for elliptic inverse problems. Appl. Numer. Math., 57:686-696, 2007.

102. Albert Tarantola. Inverse problem theory and methods for model parameter estimation. SIAM, 2005.

103. Tijmen Tieleman and Geoffrey Hinton. Lecture 6.5-rmsprop: Divide the gradient by a running average of its recent magnitude. COURSERA: Neural Networks for Machine Learning, 4, 2012.

104. Nilesh Tripuraneni, Mitchell Stern, Chi Jin, Jeffrey Regier, and Michael I Jordan. Stochastic Cubic Regularization for Fast Nonconvex Optimization. arXiv preprint arXiv:1711.02838, 2017. 
105. K. van den Doel and U. M. Ascher. On level set regularization for highly ill-posed distributed parameter estimation problems. J. Comp. Phys., 216:707-723, 2006.

106. K. van den Doel and U. M. Ascher. Dynamic level set regularization for large distributed parameter estimation problems. Inverse Problems, 23:1271-1288, 2007.

107. K. van den Doel and U. M. Ascher. Dynamic regularization, level set shape optimization, and computed myography. Control and Optimization with Differential-Algebraic Constraints, 23:315, 2012.

108. Kees Van Den Doel, Uri Ascher, and Eldad Haber. The lost honour of $\ell_{2}$-based regularization. Radon Series in Computational and Applied Math, 2013.

109. C. Vogel. Computational methods for inverse problem. SIAM, Philadelphia, 2002.

110. w. Rundell and H. W. Engl. Inverse problems in medical imaging and nondestructive testing. Springer-Verlag New York, Inc., 1997.

111. Chien-Chih Wang, Chun-Heng Huang, and Chih-Jen Lin. Subsampled Hessian Newton methods for supervised learning. Neural computation, 2015.

112. Peng Xu, Farbod Roosta-Khorasani, and Michael W Mahoney. Newton-type methods for non-convex optimization under inexact hessian information. arXiv preprint arXiv:1708.07164, 2017.

113. Peng $\mathrm{Xu}$, Farbod Roosta-Khorasani, and Michael W Mahoney. Second-order optimization for non-convex machine learning: An empirical study. arXiv preprint arXiv:1708.07827, 2017.

114. Peng Xu, Jiyan Yang, Farbod Roosta-Khorasani, Christopher Ré, and Michael W. Mahoney. Sub-Sampled Newton Methods with Non-Uniform Sampling. In Advances In Neural Information Processing Systems (NIPS), pages 2530-2538, 2016.

115. Haishan Ye, Luo Luo, and Zhihua Zhang. Revisiting sub-sampled newton methods. arXiv preprint arXiv:1608.02875, 2016.

116. Z. Yuan and H. Jiang. Quantitative photoacoustic tomography: Recovery of optical absorption coefficient maps of heterogeneous media. Applied physics letters, 88(23):231101-231101, 2006.

117. Matthew D Zeiler. Adadelta: an adaptive learning rate method. arXiv preprint arXiv:1212.5701, 2012. 